\title{
Comparison between a new holographically generated complex filter and the binary phase filter for depth of field extension
}

\author{
Frédéric Diaz ${ }^{* a, b}$, François Goudail $^{\mathrm{b}}$, Brigitte Loiseaux ${ }^{\mathrm{a}}$, Jean-Pierre Huignard ${ }^{\mathrm{a}}$ \\ ${ }^{a}$ Thales Research \& Technology, RD 128, 91767 Palaiseau Cedex, France \\ ${ }^{\mathrm{b}}$ Laboratoire Charles Fabry de 1'Institut d'Optique, RD 128, 91127 Palaiseau Cedex, France \\ *frederic.diaz@thalesgroup.com
}

\begin{abstract}
To improve the depth of field in imaging systems, we propose a new method for designing pupil filters with the classical approach used for computer-generated holograms. This method allows us to calculate a complex amplitude/phase filter in order to obtain a desired distribution of intensity along the optical axis, and thus the desired depth of field.

We will compare our complex filter with binary-phase filters, which are one of the different methods already investigated to improve the depth of field in imaging applications. This study will reveal that the complex filter is an interesting alternative for applications where very low fluctuations of the amplitude distribution along the optical axis are required. It is indeed as energy-efficient as a pure phase filter even with a non negligible absorption. It also ensures to precisely tailor the shape of the focal line of an imaging lens, as the decrease of intensity is sharper outside the regions of interest than with the binary-phase filter. Moreover, it also benefits from lower sidelobes. With these characteristics, this new complex filter will be suitable particularly for 3D imaging applications.
\end{abstract}

Keywords: pupil filter, binary-phase filter, depth of focus, computer generated hologram

\section{INTRODUCTION}

In classical imaging systems, an object or a scene is imaged by an optical system on a sensor which gives the final images. To optimize the required luminous flux, this involves a large aperture and thus reduces the depth of field or introduces chromaticism and thermal defocus, especially with infrared cameras. All these issues could be solved by a common solution : increase the depth of focus (DOF) while maintaining the transverse resolution. To do so, we consider the introduction of a filter into the optical system.

To increase the DOF, the simplest filters are probably amplitude filters such as annular aperture [1]. Continuous phase filters such as axicons and axilens [2-4], and binary-phase filters [5-7] can also enhance the DOF. They are usually considered as energy-efficient compared to amplitude filters. However, in DOF extension, the relevant parameter is not the total transmitted energy, but the "useful" one, that is, the energy concentrated in the focal line. With this criterion, amplitude/phase (complex) filters may have better performance. Besides, some complex filters have already been designed [8-10].

Our goal is to produce a light distribution as uniform as possible along the focal line. To reach such a goal, we will compare two types of methods: a classical one based on binary-phase filter (BPF), and a new approach based on a holographically generated complex filter (HGCF), which allows a precise tailoring of the focus region.

\section{BINARY PHASE FILTER}

The BPF is one of the mostly used phase filters to increase the DOF. It is composed of an array of $n$ concentric rings whose phases are alternatively 0 and $\pi$. Their outer radii are denoted $r_{1}, r_{2}, \ldots, r_{n-1}$, and are normalized with respect to the pupil radius (Fig. 1a). Assuming that the aperture is small compared to the focal length, we obtain the following normalized amplitude distribution near the optical axis generated by a lens and a BPF:

Three-Dimensional Imaging, Visualization, and Display 2009 ,

edited by Bahram Javidi, Jung-Young Son, Manuel Martinez-Corral, Fumio Okano, Wolfgang Osten, Proc. of SPIE Vol. 7329, 73290B - (C) 2009 SPIE · CCC code: 0277-786X/09/\$18 - doi: 10.1117/12.821177

Proc. of SPIE Vol. 7329 73290B-1 


$$
A(\rho, z)=\exp (j k z) / z \times \exp \left(j k \rho^{2} / 2 z\right) \times \int_{0}^{R} P\left(\rho_{P}\right) \rho_{P} J_{0}\left(k \rho \rho_{P} / z\right) \exp \left[j k \rho_{P}{ }^{2}(1 / z-1 / f) / 2\right] d \rho_{P}
$$

with $\lambda$ the wavelength, $k=2 \pi / \lambda, f$ the focal length and $R$ the radius of the lens, $\rho$ and $z$ the transverse and axial coordinates, $\rho_{P}$ the transverse coordinate in the pupil plane, $J_{0}$ the 0 -order Bessel function, and $P\left(\rho_{P}\right)$ the complex amplitude of the BPF, equal to \pm 1 .

Using Eq. (1), one can perform an exhaustive search to find the parameters $r_{i}$ that provides the maximal mean intensity in the DOF region for a given DOF, but there are no analytical method to obtain these optimal parameters. We compute the DOF and the mean intensity in the DOF region of all 3-ring filters by varying the radii of each ring with a step of 0.001 , using a lens with a focal length of $160 \mathrm{~mm}$ and an aperture of $16 \mathrm{~mm}$, at a wavelength of $1064 \mathrm{~nm}$, with intensity fluctuations below $10 \%$. We found that the 3-ring filter which can achieve the maximal DOF is the filter whose parameters are $r_{1}=0.345$ and $r_{2}=0.618$ (Fig. 1).
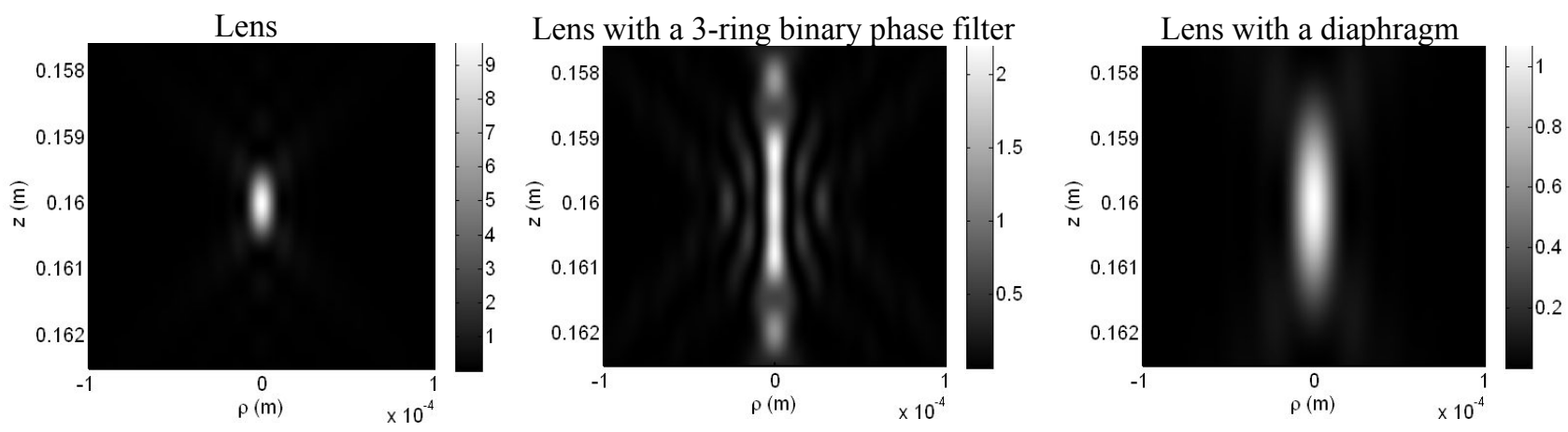

Fig. 1. Comparison between the point spread functions of a lens, a 3-ring binary phase filter with fluctuations below $10 \%$, and a lens with a diaphragm

The 3-ring binary phase filter has a DOF of $2.4 \mathrm{~mm}$, which is 3 times larger than the DOF of the lens, while the mean intensity in the DOF region is divided by a factor 4 . The transverse resolution is maintained since the full width at half maximum (FWHM) of the single lens is $110 \mu \mathrm{m}$ while the FWHM of the binary phase filter is $90 \mu \mathrm{m}$.

To compare, if one wanted to produce a DOF 3 times larger with a lens, the aperture should be reduced by a factor $\sqrt{3}$, but the FWHM would be increased by a factor $\sqrt{3}$, and the mean intensity would be divided by 9 , a factor 3 coming from the loss of energy due to the smaller aperture, and a factor 3 coming from the spreading of the intensity along the DOF region. Thus, the 3-ring binary phase filter gives better performance than a lens with a diaphragm.

\section{HOLOGRAPHICALLY GENERATED COMPLEX FILTER}

As an original method, we now propose to design a complex filter with the classical approach used for computergenerated holograms. Such design relies on a point by point construction method : to generate a focal line, one can calculate an amplitude distribution in the pupil plane which generates along the optical axis the desired amplitude distribution. If one wants to obtain, at the coordinate $z_{1}$ of the optical axis, a desired amplitude $D_{1}$, this corresponds to a given amplitude distribution on the pupil. If one now wants to obtain, at the coordinate $z_{2}$ of the optical axis, another desired amplitude $D_{2}$, this corresponds to another amplitude distribution on the pupil. In order to obtain both desired amplitudes on the axis at the given coordinates, the corresponding amplitude distribution in the pupil is the "sum" of the previous amplitude distributions.

Indeed, the amplitude distribution in the pupil associated to a point at the coordinate $z_{i}$ in the focal line is equal to 


$$
T_{i}(\rho)=A_{i} \exp \left(-j k \rho^{2} / 2 z_{i}\right) / z_{i}
$$

which is in fact the complex transmittance of a lens with a focal length $z_{i} . A_{i}$ represents the own amplitude of each point. Illuminated by a plane wave, this lens generates an amplitude distribution along the optical axis equal to:

$$
A_{z}\left(z, A_{i}, z_{i}\right)=A_{i} \times A_{p t}\left(z, z_{i}\right) \times \exp \left(j \varphi_{p t}\left(z, z_{i}\right)\right)
$$

with $A_{p t}\left(z, z_{i}\right)=2 \sin \left[k\left(1 / z-1 / z_{i}\right) R^{2} / 4\right\rfloor /\left[k\left(z_{i}-z\right)\right]$ and $\varphi_{p t}\left(z, z_{i}\right)=k\left(z-z_{i}\right)+k\left(1 / z-1 / z_{i}\right) R^{2} / 4$.

The amplitude distribution of the filter associated with all the points is thus

$$
T(\rho)=\sum_{i} T_{i}(\rho)
$$

By taking $A_{i}$ equal to $D_{i}$, one will not obtain the amplitude $D_{i}$ at the coordinate $z_{i}$ because the amplitude distribution along the optical axis associated to one point is generally not null at the coordinate of the others points. In order to cancel the influence of the amplitude associated to one point over the others, one can choose points which cancel the $\sin$ term in Eq. 3. Unfortunatly, theses points are not close enough, and we observed large fluctuations of intensity between them which are incompatible with our goal.

In order to obtain low variations, we consider the influence of the amplitude associated to one point onto the others, and create an interaction matrix $\mathrm{Z}$ defined as follow :

$$
Z_{i, j}=A_{z}\left(z_{j}, 1, z_{i}\right)
$$

It contains the value of amplitude distribution associated to each point $i$, with $A_{i}=1$, at the coordinates $z_{j}$.

The coefficients $A_{i}$ so that, $\forall i, A_{z, t o t}\left(z_{i}\right)=D_{i}$, are calculated by solving the following set of linear equations:

$$
\vec{D}=Z \times \vec{A}
$$

where vector $\vec{D}$ contains the $D_{i}$ and vector $\vec{A}$ contains the $A_{i}$. The values of $A_{i}$ are obtained by inverting the matrix $Z$, and the complex transmission of the obtained HGCF is:

$$
T(\rho)=\frac{\sum_{i} T_{i}(\rho)}{\max _{\rho}\left|\sum_{i} T_{i}(\rho)\right|} \exp \left(j k \rho^{2} / 2 f\right)
$$

where the phase already introduced by the lens has been removed and the transmittance normalized so that its maximal modulus is 1 . Since we consider spatially incoherent imaging, the interesting value is the intensity distribution along the optical axis:

$$
I_{z}(z) \propto\left|\sum_{i} A_{i} \times A_{p t}\left(z, z_{i}\right) \times \exp \left(j \varphi_{p t}\left(z, z_{i}\right)\right)\right|^{2}
$$

While the function $A_{p t}\left(z, z_{i}\right)$ has relatively slow variations, the $\exp \left(j \varphi_{p t}\left(z, z_{i}\right)\right)$ phase terms introduce fast and large intensity fluctuations between the point sources. These terms can be removed by choosing point sources satisfying the relation $\varphi_{p t}\left(z_{i}, f\right)=2 \pi p_{i}$ with $p_{i}$ integer numbers. These points are located at the coordinates:

$$
z_{i}=\frac{1}{2}\left(f+R^{2} /(4 f)+p_{i} \lambda\right) \pm \frac{1}{2} \sqrt{\left(f+R^{2} /(4 f)+p_{i} \lambda\right)^{2}-R^{2}}
$$

If all the point sources satisfy such property, the intensity distribution along the optical axis can now be written:

$$
I(z) \propto\left|\sum_{i} A_{i} \times A_{p t}\left(z, z_{i}\right)\right|^{2}
$$


By taking point sources close enough to each other compared to the width of the function $A_{p t}\left(z, z_{i}\right)$, with represents the DOF of a single lens, the variations of intensity can be reduced below $1 \%$. The mean intensity in the DOF region can be further improved by optimizing their positions $z_{i}$ and desired amplitudes $D_{i}$. We also use two extra point sources, before and after the DOF region, with low desired amplitudes, to decrease faster the intensity outside the DOF region. We noticed that this also increases the intensity in the DOF region. Performing a numerical optimization, we managed to increase the intensity in the DOF region up to a factor 3 compared to the non-optimized HGCF.

Using this method, we achieve to obtain a DOF of $1.6 \mathrm{~mm}$ with variations lower than 1\% (Fig. 2).

a) PSF

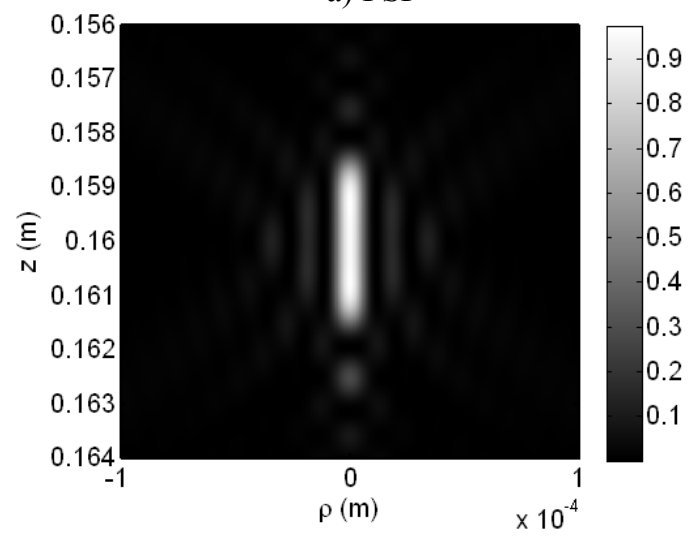

b) Profile of the HGCF
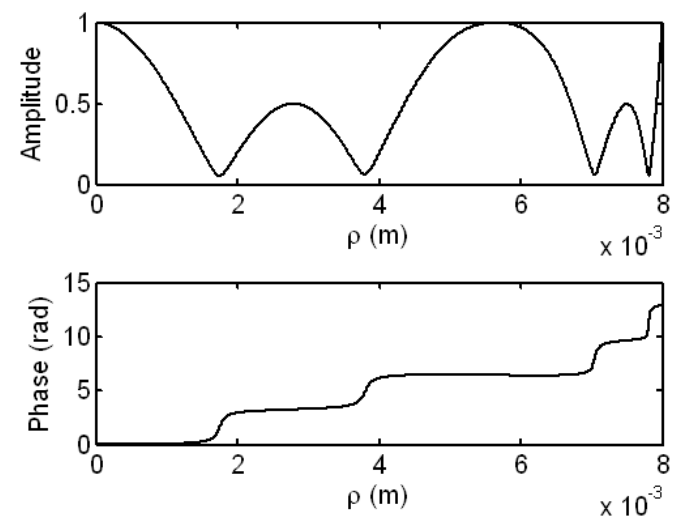

Fig. 2. PSF of the HGCF with a DOF of $1.6 \mathrm{~mm}$ and fluctuations below $1 \%$, and its corresponding profile

\section{COMPARISON BETWEEN THE BINARY PHASE FILTER AND THE HOLOGRAPHICALLY GENERATED COMPLEX FILTER}

\subsection{Comparison of the PSF}

In order to compare the performance of the PSF of the HGCF with the BPF, we took the BPF giving the better mean intensity on the DOF region for a DOF of $1.6 \mathrm{~mm}$ with fluctuations below $1 \%$. Its parameters are

$$
r_{1}=0.26 \quad r_{2}=0.39 \quad r_{3}=0.54 \quad r_{4}=0.58 \quad r_{5}=0.84 \quad r_{6}=0.96
$$

Fig. 3 shows the PSF of the lens, the BPF and the HGCF. Their performance are comparable, although the HGCF has a averaged transmission of $30 \%$. This drop of transmission is compensated by a better concentration of energy in the DOF region, thanks to the contribution of amplitude part of the filter. Moreover, with the HGCF, it is possible to achieve an accurate design of the DOF region which is directly linked to the choice of point sources. In our example, the DOF region is centred on the focal point, whereas the DOF region of the BPF is asymmetrically distributed between $z=158.4 \mathrm{~mm}$ and $160 \mathrm{~mm}$. Further advantages of the HGCF are the sharper decrease of the intensity at the limit of the DOF region, lower sidelobes and the better invariance of the transverse spot size with respect to $z$ inside the DOF region. 
a) Lens
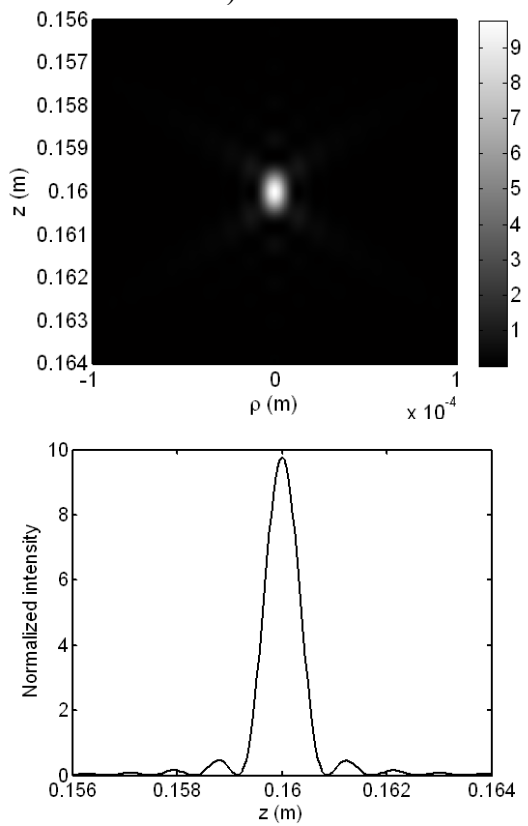

b) $\mathrm{BPF}$
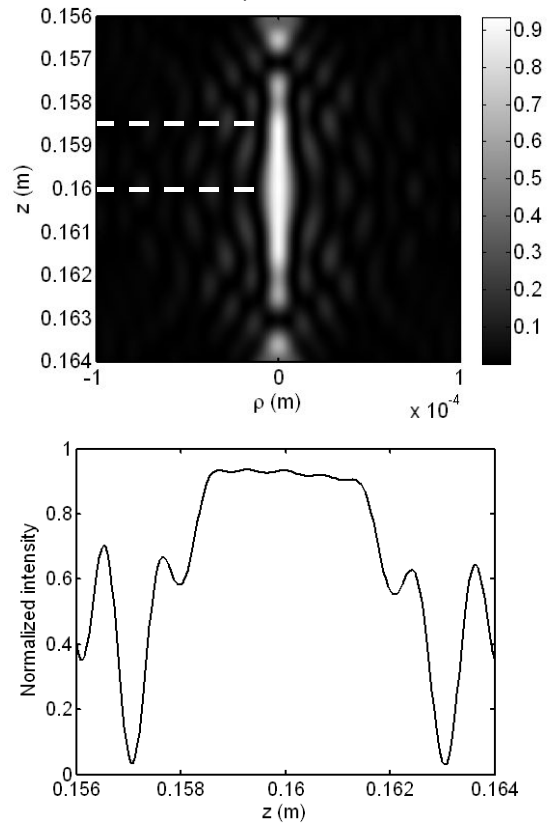

c) $\mathrm{HGCF}$
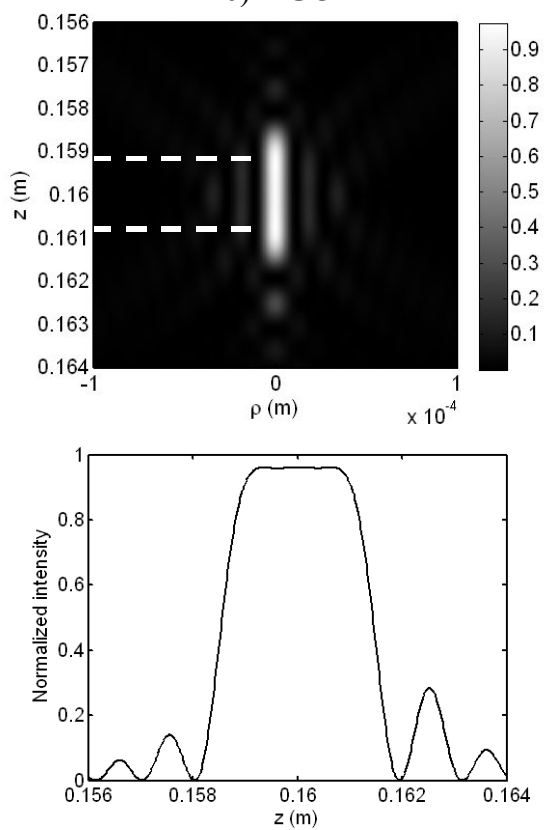

Fig. 3. PSF of the lens, the BPF and the HGCF for a DOF of $1.6 \mathrm{~mm}$ and fluctuations below $1 \%$. The DOF region where the variations are below $1 \%$ are delimited with the dotted lines

\subsection{Phase and amplitude profiles}

The phase distributions of the BPF and the HGCF plotted in Fig. 4 show great similarities, as the HGCF presents 4 closeto- $\pi$ phase jumps. However, we have checked that setting the transmission of the HGCF to 1 on the whole pupil does not provide good results. The amplitude modulation is required to yield an optimal result.

a) Profile of the BPF
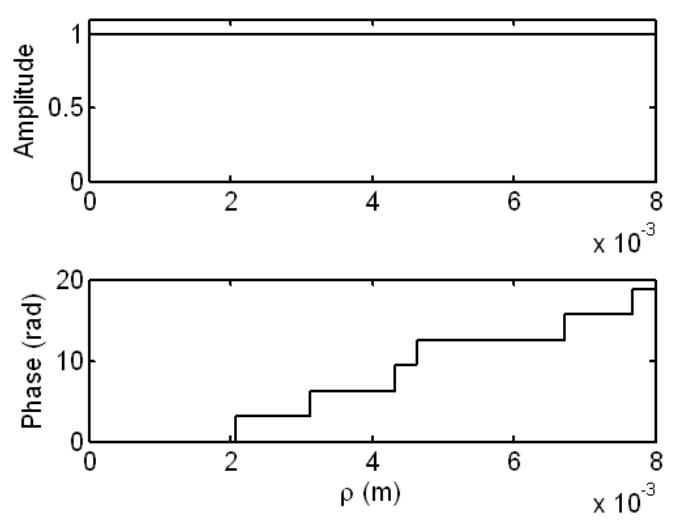

b) Profile of the HGCF
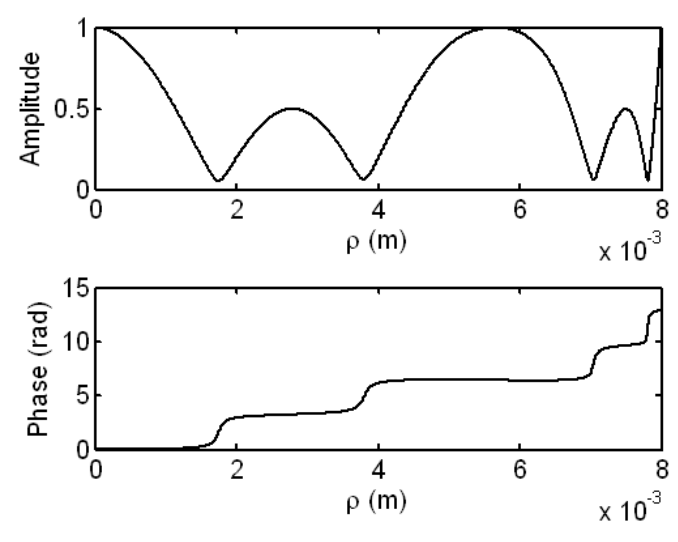

Fig. 4. Profiles of the amplitude and phase of the BPF and the HGCF.

\subsection{Spectral behaviour}

In conventional optically generated off axis holograms, the spacing of the interference fringes is comparable to the wavelength, making the hologram output very sensitive to variations of the illumination wavelength. With the HGCF, the scale of the spatial variations of the phase and the amplitude is very large compared to the wavelength, as can be seen 
in Fig. 2. Consequently, the amplitude and phase profile of the HGCF should not depend on the construction wavelength. To show this, we have plotted in Fig. 5 the amplitude and the phase profiles of two HGCF: the HGCF obtained for a DOF of $1.6 \mathrm{~mm}$, with a focal length $f=160 \mathrm{~mm}$, an aperture radius $R=8 \mathrm{~mm}$, at wavelength of $\lambda=1064 \mathrm{~nm}$, which is in fact already represented in Fig. 2 and Fig. 4, and the HGCF for the same focal length and aperture radius at a wavelength of $\lambda=632 \mathrm{~nm}$, which is designed to produces the same defocus given by the following defocus parameter $\psi=\frac{\pi R^{2}}{\lambda}\left(\frac{1}{f}-\frac{1}{z}\right)=5.9$, corresponding to a DOF equal to $0.95 \mathrm{~mm}$ at a wavelength of $\lambda=632 \mathrm{~nm}$. As one can see, the profile are almost equal for the two different construction wavelengths. That is to say that the phase and the amplitude does not depend on the construction wavelength.
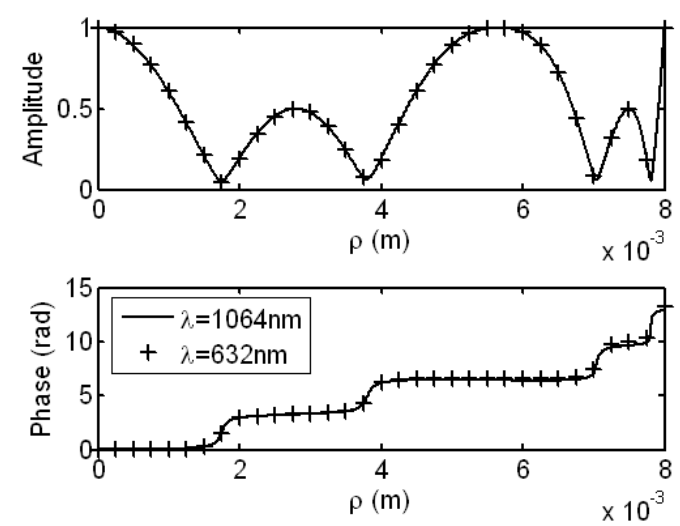

Fig. 5. Phase and transmission profile of two HGCF designed to obtain a DOF corresponding to $\psi=5.9$, at two different wavelengths

We also plotted in Fig. 6 the incoherent response of the HGCF that gives a DOF of $1.6 \mathrm{~mm}$ at a wavelength $\lambda=1064 \mathrm{~nm}$ at different illumination wavelengths, while neglecting the materials dispersion of the filter. Even if the performance decreases, we see that they remain quite good for a range of a hundred of nanometers. Thus this filter is usable with non monochromatic but relatively narrow band illumination. Moreover, one can see that the HGCF is less sensitive to the illumination wavelength than the BPF. 
$\mathrm{BPF}$
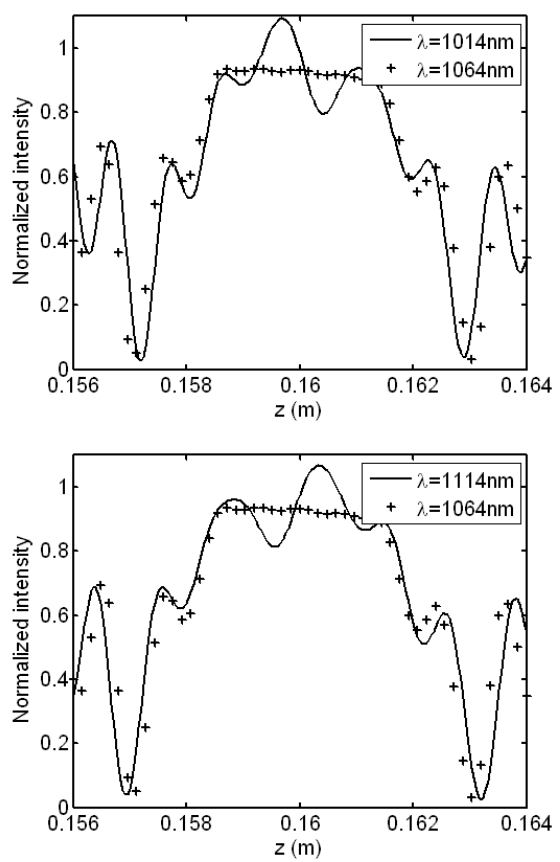

HGCF
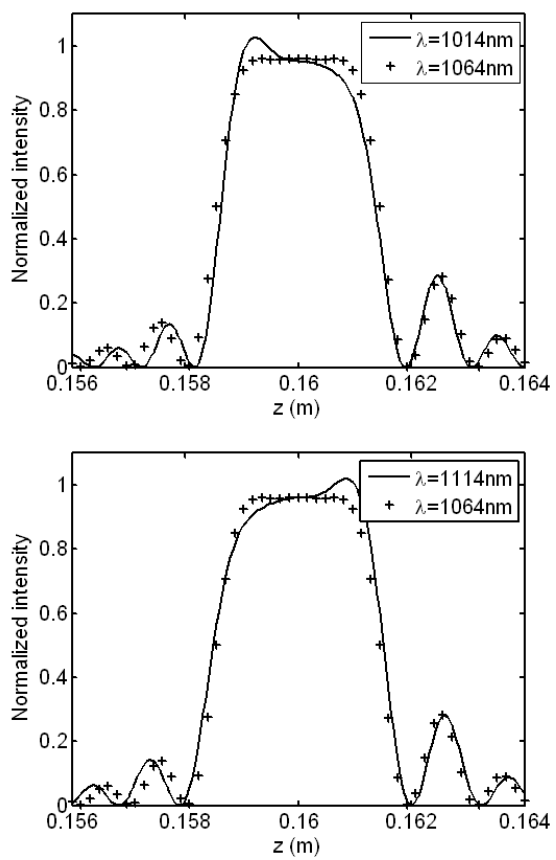

Fig. 6. PSF of the BPF and the HGCF with different illumination wavelengths

On the other hand, for operation in a larger spectral band, it will be necessary to use materials whose dispersion compensates the phase mismatch in the desired spectral range. Such materials exists or can be obtained by subwavelength structured materials as described by [11].

\section{CONCLUSION}

We proposed a new holographic method for the design of an amplitude/phase filter convenient for depth of focus extension, that we called HGCF. This filter is an alternative to the binary phase filter for applications when very low fluctuations of the amplitude distribution along the optical axis are required and when the shape of the focal line has to be precisely tailored. It also benefits from lower sidelobes and a better invariance of the point spread function with respect to $z$ inside the DOF region. Such complex filter which are as efficient as pure phase filter may apply for coded aperture imaging systems without the implementation of a post-processing, thanks to its quasi-invariant PSF.

\section{ACKNOWLEDGEMENTS}

This work benefits from partial support of the ANRT French Administration.

\section{REFERENCES}

[1] W. T. Welford, "Use of annular aperture to increase focal depth", J. Opt. Soc. Am. A 50, 749-753 (1960)

[2] J. H. McLeod, "The axicon: a new type of optical element”, J. Opt. Soc. Am. 44, 592-597 (1953)

[3] J. Sochacki, S. Bara, Z. Jaroszewicz, and A. Kolodziejczyk, "Phase retardation of the uniform-intensity axilens", Opt. Lett., 17, 7-9 (1992)

[4] E. Marom, D. Mendlovic, N. Konforti, J. Katz and C. Tan, "Diffractive optic elements for forming scanning beams", Proc. of SPIE, 3010, 30-37 (1997) 
[5] H. Wang, and F. Gan, "High focal depth with a pure-phase apodizer", Appl. Opt. 40, 5658-5662 (2001)

[6] L. Liu, C. Liu, W. C. Howe, C. J. R. Sheppard, and N. Chen, "Binary-phase spatial filter for real-time swept-source optical coherence microscopy”, Opt. Lett. 32, 2375-2377 (2007)

[7] L. Liu, F. Diaz, L. Wang, B. Loiseaux, J-P. Huignard, C. J. R. Sheppard, and N. Chen, "Superresolution along extended depth of focus with binary-phase filters for the Gaussian beam”, J. Opt. Soc. Am. A 25, 2095-2101 (2008)

[8] M. Yun, L. Liu, J. Sun, and D. Liu, "Three-dimensional superresolution by three-zone complex pupil filters", J. Opt. Soc. Am. A 22, 272-277 (2005)

[9] S. Förster, H. Gross, F. Höller and L. Höring, "Extended depth of focus as a process of pupil manipulation", Proc. of SPIE, 5962, 44-53 (2005)

[10] S. Bagheri and B. Javidi, "Extension of depth of field using amplitude and phase modulation of the pupil function", Opt. Lett., 33, 757-759 (2008)

[11] French patent $n^{\circ} 0707562$. 